\title{
Comparison of De-noising Algorithms Technique
}

\author{
Ogunsanwo Gbenga Oyewole ${ }^{1}$, Goga Nicholas ${ }^{1}$, Awodele Oludele ${ }^{1} \&$ Okolie Samuel $^{1}$ \\ ${ }^{1}$ Computer Science Department, Babcock University, Ilisan Remo, Nigeria \\ Correspondence: Dr. Ogunsanwo Gbenga Oyewole, Computer Science Department, Babcock University, Ilisan \\ Remo, Nigeria. E-mail: ogunsanwogo@gmail.com
}

$\begin{array}{lc}\text { Received: April 9, } 2018 & \text { Accepted: May 25, } 2018 \quad \text { Online Published: June 11, } 2018 \\ \text { doi:10.5539/nct.v3n1p26 } & \text { URL: https://doi.org/10.5539/nct.v3n1p26 }\end{array}$

\begin{abstract}
The concept of noise appears during the process of gathering the image into digital form: that is when the image is being created and it may also be introduced when the image is being transmitted. The presence of the noise usually degraded the quality of the image. De noising algorithms were employed in order to advance the value of the image. This paper tries to compare linear and non linear filtering algorithm. This study adopted image processing techniques to process 600 images dataset acquired from 60 different signers using vision based method. The acquired images were de-noised using Gaussian filter and Median filter algorithms. The outcomes of the two de-noising algorithms were compared using Peak Signal to Noise Ratio (PSNR) and Mean Square Error (MSE). The results of processed images for de-noising algorithms show that Median filter had higher PSNR of 47.7 than the Gaussian filter of 31.79, and lower MSE of 1.11 than Gaussian filter of 43.4.It was also ascertained that de-noised images with non-linear median filter had better quality than images de-noised by linear Gaussian filter.
\end{abstract}

Keywords: noise, linear filtering algorithm techniques, non linear filtering algorithm techniques, gaussian filter, median filter

\section{Introduction}

Image de-nosing is the process of filtering or removing noise from an image so that the standard of the image will be okay or the signal noise ratio (SNR) will be high (Liping \& Jinfang, 2016). Image de-noising is very crucial or important task in image processing for the analysis of images. The major goal in image de-noising is to remove the noise from the image in such a way that the original image is distinct. In modern digital image processing, data de-noising is a common problem and it is now a major concern of diverse application areas (Astola \& Kuosmanen, 1997). Image de-noising is an important stage in image processing in order to improve the performance of the recognition system. (Ogunsanwo, Goga, Awodele, \& Okolie, 2018) designitng any recognition system. There are numerous systems for filtering noise from images (Astola \& Kuosmanen, 1997). There are two types of spatial filtering models which are; linear model and non-liner model. Progressive works have been done by many researchers, most especially in the area of image processing, this work has majorly focused on discovery appropriate alternatives to the linear filter that are robust or opposed to the occurrence of impulsive noise. Among these, the discovery that has received great awareness is median filters. (Juan \& Gonzalo 2002)

There are many issues associated with filtering method which are the processing speed, inability to preserve edges of the images in an efficient manner; that is, the edges, which are recognized as discontinuities in the image, are tarnished (Pawan et al, 2010; Govindaraj \& Sengottaiyan, 2013). An effective de-noising system must able to recover image details when the image is greatly filled with noise (Astola \& Kuosmanen, 1997). Since image de-noising model are categorized into two types that is linear model and non-linear model. In this paper we try to experiment one example from each of the model in order to ascertain their efficiency in collaboration with the general believe found in the literature. Gaussian filtering algorithm was picked for linear while median filtering was selected for non linear and their efficiency were determined using Peak Signal to Noise Ratio (PSNR) and Mean Square Error (MSE) The PSNR and MSE are useful for calculating the development in de-noisedimages (Afrah, Firas, \& Atilla, 2017). The experiment was carried out using matlab software. 


\section{Methodology}

\subsection{Raw Data Acquisition}

Data from 60 subjects (signers) were collected and utilized for the study. Each subject generated 10 samples of each static one to ten Figures (600 total samples). The data were subsequently used to evaluate the performance of both models. In order to acquire data, the vision-based sign extraction method was used. This was achieved by fetching an input image based on a camera. Figure 1 shows the samples of gestures created for the dataset. To create a gesture system database, the gestures were selected along with their connotations, in which each gesture contained various samples to increase the accuracy of the system. Vision-based method was chosen because it is user friendly and popularly used in sign recognition systems. A fixed camera in front the signers were used to capture the sign gesture. Posture features of the fingers and palms were further extracted. After the data were collected, the images passed through different stages of image processing technique in order to enhance the quality of the image

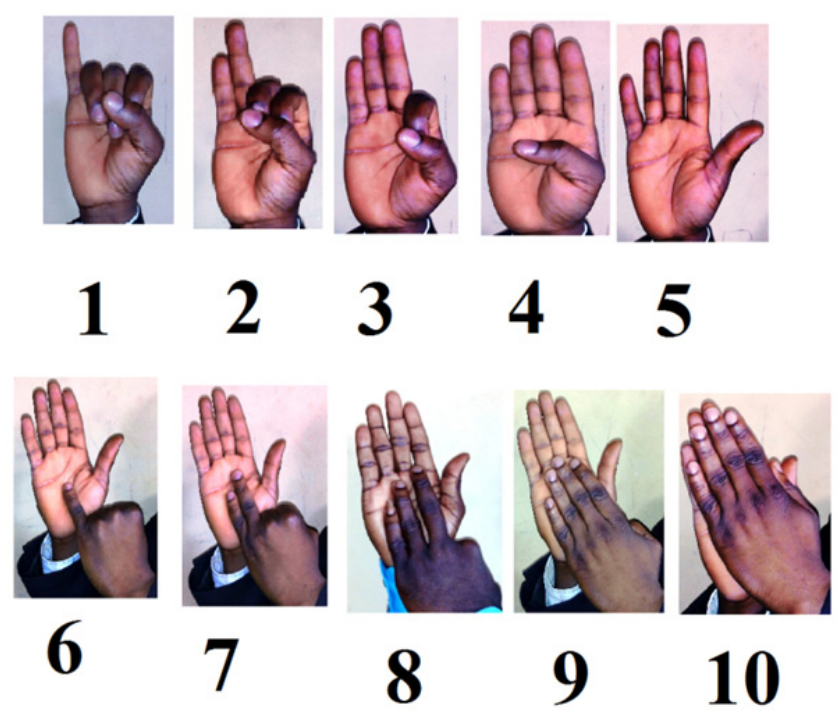

Figure 1. SamplesofDataset Used in the Research

\subsection{Image Pre-Processing and De-noising}

Image pre-processing was employed in the research to enhance the gathered data. The image pre-processing procedure involved converting coloured images to grayscale, resizing and de-noising. In order to make the image scale invariant, the pre-processed image were cropped to segment the background and concentrate only on the palms and fingers. To obtain this, the original images were converted to gray scale images using threshold method. The cropped gray scale images were further resized 50 × 80 pixels making the sign image scale independent. Thereafter, Gaussian and Median filtering techniques were used to remove the interference noise from the image dataset, while the performance of these two filtering algorithms was evaluated using Peak Signal to Noise Ratio (PSNR) and Mean Square Error (MSE). For one dimension Gaussian function is expressed as:

$$
G(j)=\frac{1}{\sqrt{2 \prod \sigma}}\left[e^{-I^{2}} / 2 \sigma^{2}\right]
$$

While the two dimensional can be expressed as:

$$
\mathrm{G}(\mathrm{j}, \mathrm{k})=\frac{1}{\sqrt{2 \pi \sigma}} e^{-\left(\frac{j^{2}+k^{2}}{2 \sigma^{2}}\right)}
$$

the standard deviation of the Gaussian distribution is represented by $\sigma$, the variance is represented by $\sigma^{2}$

the distance from the source in the horizontal axis is represented by $\mathrm{j}$, 
the distance from the source in the vertical axis is represented by $\mathrm{k}$,

$(\mathrm{j}, \mathrm{k})$ represents specific pixel position in the image.

Using non-linear Median Filter.

How median filtering algorithm works

Median filtering algorithm functions by considering each pixel in an image and examine its close neighbour to decode if it represents the surrounding or not. The pixel value will be replaced with median of that value. The median is derived by first arranging all the pixel values from the environment into numerical form, ascending order and thereafter with medina pixel will be selected from the pixel under consideration. If the neighbourhood under consideration contain even number of pixel, the average of this middle pixel value is used.

$$
\mathrm{a}(\mathrm{w})=\operatorname{median}((\mathrm{b}(\mathrm{w}-\mathrm{T} / 2), \mathrm{b}(\mathrm{w}-\mathrm{T} 1+1), \ldots, \mathrm{b}(\mathrm{w}), \ldots, \mathrm{b}(\mathrm{w}+\mathrm{T} / 2))
$$

where w represent the window's sixe of the median filter.

$\mathrm{b}$ is the dimension of the neighbourhood

$\mathrm{T}$ is the neighbouring pixel under consideration

\section{Result and Discussion}

\subsection{Gaussian Enhancement on the Acquired Sign Gesture Images}

The acquired images had some noise, which had been introduced during capturing, the noise were introduced as a result of shadows from flash light of the digital camera used. To remove the noise, Gaussian filter was used to enhance the images, the Yoruba signs gestures were selected one after the other from the source folder. The Gaussian function was curled with the images. The output of this was a blur enhanced image that is free from noise. These processed images were stored in another folder that has been specified as the destination for the pre-processed images.

In order to enhance the images, the Gaussian radius and sigma value that was used to determine the level of enhancement was supplied. The level of blurring that will occur during the processing is determined by Sigma value. The Radius was used to determine the size of the array which will hold the calculated Gaussian distribution. It should be an integer. The larger the Radius the slower the operations become. Figure 2 to Figure 5 show the filtered sign gesture images at a Gaussian radius of 20 .

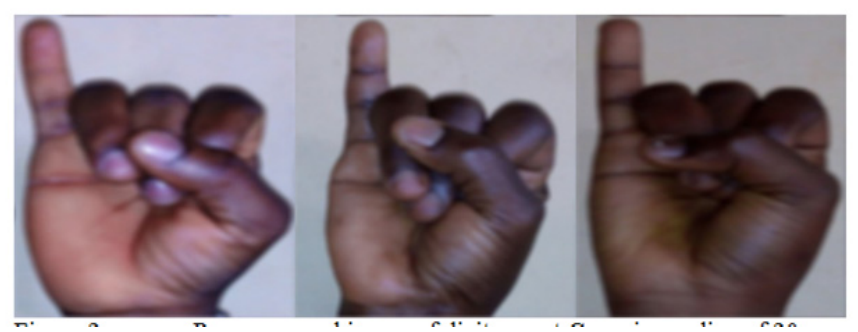

Figure 2. Pre-processed image of digit one at Gaussian radius of 20

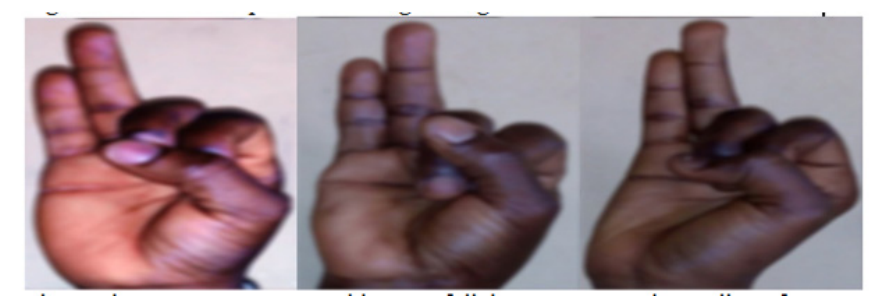

Figure 3. Pre-processed image of digit two at Gaussian radius of 20 


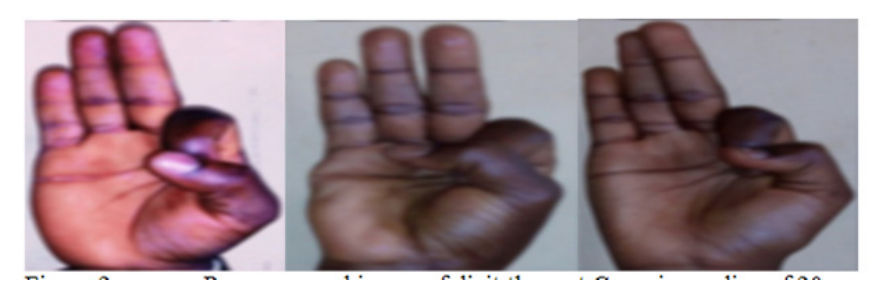

Figure 4. Pre-processed image of digit three at Gaussian radius of 20

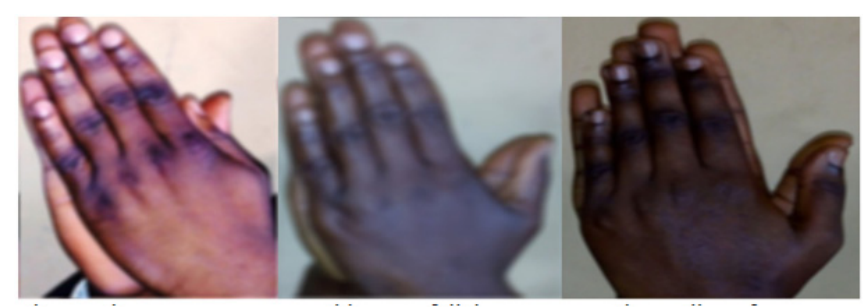

Figure 5. Pre-processed image of digit ten at Gaussian radius of 20

\subsection{Median Enhancement on the Acquired Sign Gesture Images}

Figure 6 to Figure 9 shows the result of the pre-processed sample image in Figure 1 based on median filtering. It is observed that images appeared to be cleaner than the images: which had been de-noised at Gaussian Radius of 20, this implies that the non linear filtering method of median filter performed better than the linear filtering method of the Gaussian.
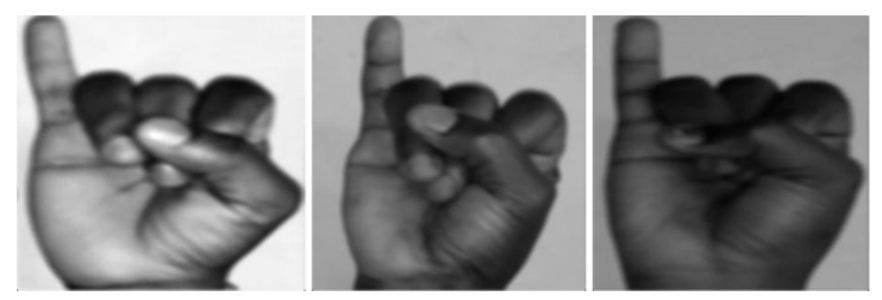

Figure 6. Pre-processed image of digit one based on Median Filtering
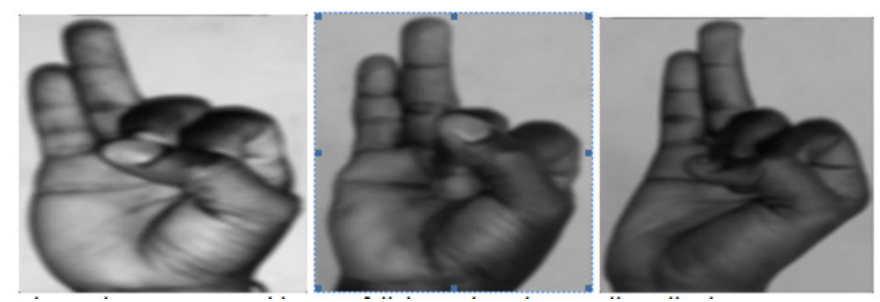

Figure 7. Pre-processed image of digit two based on Median Filtering 


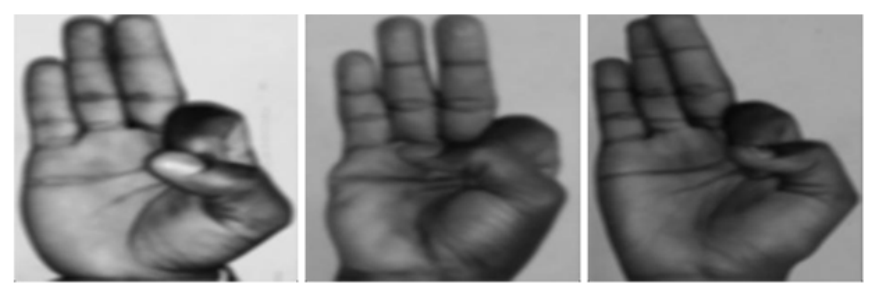

Figure 8. Pre-processed image of digit three based on Median Filtering
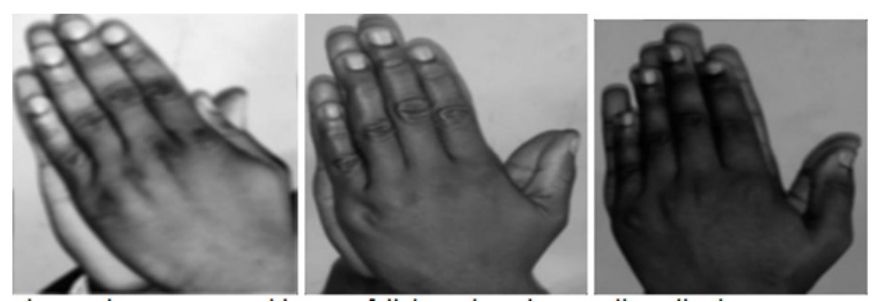

Figure 9. Pre-processed image of digit ten based on Median Filtering

\section{Evaluation of Filtering Method}

In order to ascertain the efficiency of the filtering algorithms method for de noising the sign gesture, the filtered images were compared with respect to the original images before filtering, using Peak Signal to Noise Ratio (PSNR) and Mean Square Error (MSE) as shown in Figure 10 and Figure 11 respectively. Figure 10 shows that Median filter had higher PSNR of 47.7 than the Gaussian filter with 31.79, more so, Figure 11 shows that Median filter had a lower MSE of 1.11, while Gaussian filter has a MSE of 43.4. The PSNR is measure by its value, the higher the value the better the performance of signal to noise rate in that image while in the case of MSE the lower value signify good performance. This implies that images de noised by the Median filtering method had better quality than Gaussian filtering.

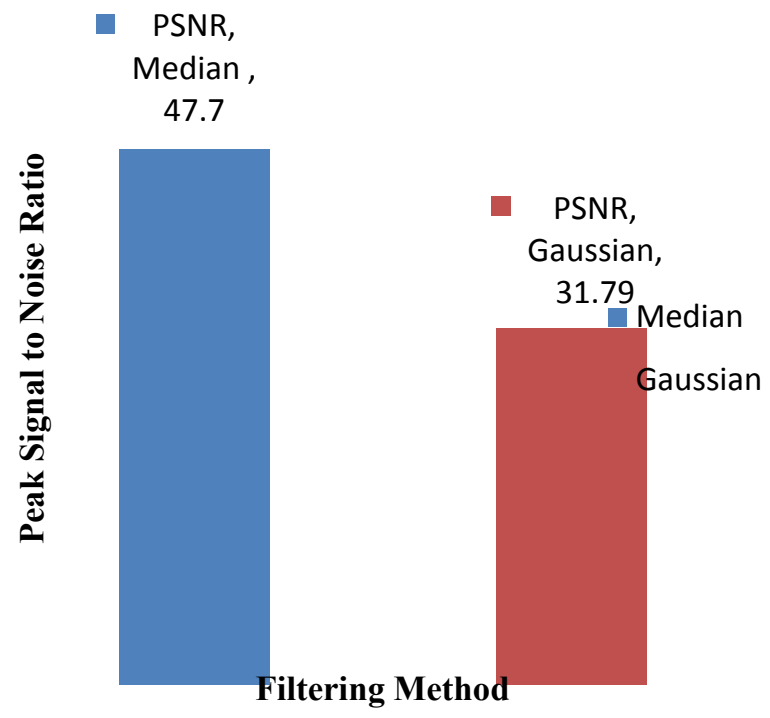

Figure 10. Comparison of Filtering Method based on PSNR 


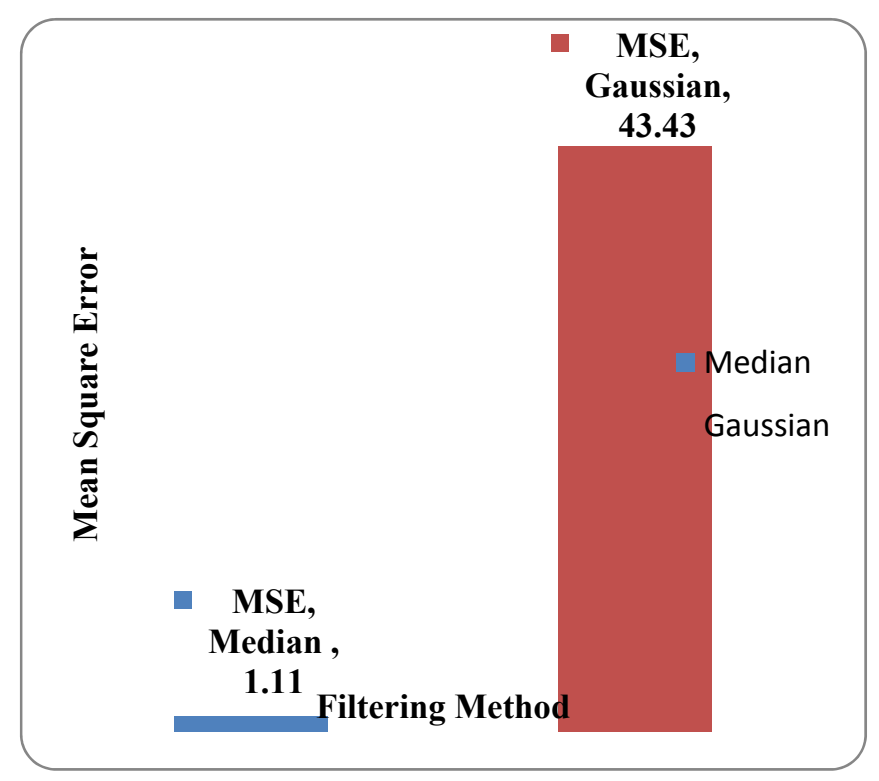

Figure 11. Comparison of Filtering Method

\section{Conclusion}

In this paper we used dataset of 600 images from sixty different signers were gathered. The images were acquired using vision based method The acquired images were first resize to make the images constituent, noise were removed using Gaussian filter and median algorithm. In order to ascertain the most efficient filtering methods out of the two adopted; their performance were examined using Peak Signal to Noise Ratio (PSNR) and Mean Square Error (MSE), which unrevealed that the non-linear median filtering performed better. In conclusion, de-noised images with non-linear median filter had better quality also has the ability to preserve edges of the images in an efficient manner than images de-noised by linear Gaussian filter. The outcome of this research hereby recommended that researchers

\section{References}

Afrah, R., Firas, M., \& Atilla, E. (2017).Image denoising by median filter in Wavelet domain The International Journal of Multimedia \& Its Applications (IJMA), 9(1). https://doi,org/10.5121/ijma.2017.9104 31

Astola, J., \& Kuosmanen, P. (1997). Fundamentals of Non-linear Digital Filtering. CRC Press, New York: Boca Raton

Govindaraj, V., \&Sengottaiyan, G. (2013). Survey of Image Denoising using Different Filters. International Journal of Science, Engineering and Technology Research (IJSETR). 2(2), 344-351.

Hasan, M. M., \& Mishra, P. K. (2010). HSV brightness factor matching for gesture recognition system. QInt. J. Image Process., 4, 456-467.

Juan, G. G., \& Gonzalo, R. A. (2002). Statistically-Efficient Filtering in Impulsive Environments: Weighted Myriad Filters EURASIP. Journal on Applied Signal Processing, 1, 4-20.

Liping, L., \& Jinfang, W. (2016). Image Processing And Recognition Algorithm For Target Tracking. International Journal On Smart Sensing And Intelligent Systems, 9(1).

Ogunsanwo, G. O., Goga, Ni., Awodele, O., \& Okolie, S. (2018). Bridging Communication Gap Among People with Hearing Impairment: An Application of Image Processing and Artificial Neural Network. International Journal of Information and Communication Sciences, 3(1), 11-18

Pawan, P., \& Sumit, S. (2010). Image De-noising by Various Filters for Different Noise. International Journal of Computer Applications, 9(4), 45-50. 


\section{Copyrights}

Copyright for this article is retained by the author(s), with first publication rights granted to the journal.

This is an open-access article distributed under the terms and conditions of the Creative Commons Attribution license (http://creativecommons.org/licenses/by/4.0/). 